Sedum of North America

Sedum of North America, North of the Mexican Plateau. By Robert T. Clausen. Pp.742. (Cornell University: Ithaca and London, 1976.) £42.25.

Sedum is the largest genus of the family Crassulaceae and consists of about 300 species, most of them in the north temperate zone but some on subtropical and tropical mountains. They are succulent plants, usually low-growing and small-leaved, and on account of their general easiness of cultivation and propagation and their numerous small starry flowers they have long been esteemed as rock-garden plants. Their garden value led an Irish botanist, Robert Lloyd Praeger, to publish in 1921 an account of the genus Sedum as found in cultivation (J.R. Hort. Soc., 46, 1-314) describing and illustrating 151 species, still a most useful work. A Swedish botanist, Harald Fröderström, published between 1930 and 1935 a survey of the genus as a whole in Acta Horti Gothoburgensis, distinguishing 327 species. Neither competes in bulk and wealth of detail with Robert T. Clausen's Sedum of North America, which devotes 742 pages to some 90 species, only 36 of them native. Unlike other workers on the genus, which he has studied intensively since 1935, Professor Clausen has been able to examine wild populations of almost every North American species and has supplemented his field surveys by experimental culture at, Cornell University. Thus he has been able to separate environmental modifications from genetically fixed characters.

The result is this comprehensive monograph admirably illustrated with various sketch maps and with line drawings of all the species by Elfriede Abbe, who earlier provided those in Clausen's Sedum of the Trans-Mexican Volcanic Belt (1959). That work set out to be "an exposition of taxonomic methods", not simply a concise working account of 28 Mexican species, and provided a prolixity of detail irrelevant for almost all its users. In Sedum of North America, because the area covered is about 216 times larger (though with almost the same number of species), the same treatment has resulted in this huge and expensive work. It raises the question as to whether the printing (as distinct from the research and recording) of so many measurements in the diffuse papercovering style of this book is justifiable nowadays, since the information relevant for most purposes could have been presented in a volume of less than half the size and half the price. It thus suffers from a defect produced by its major virtue. Having worked so long, so thor- oughly and so successfully at understanding these plants which can indeed only be properly evaluated from living material studied both in the wild and under cultivation, Professor Clausen has evidently felt he must publish everything, an honest enough procedure but costly. Thus his account of Sedum spathulifolium occupies 35 pages; he nevertheless leaves somewhat uncertain Praeger's var. purpureum, of which he has seen no material, although this is still cultivated in England.

For anyone interested in the Sedum species of North America, their characters, population variation, nomenclature, distribution and identification, this work will be indispensable, which makes its high cost all the more regrettable. Likewise regrettable is the lack of attention

\section{Statistical mechanics of liquids}

Theory of Simple Liquids. By $\mathbf{J}$. Hansen and I. R. McDonald. Pp. $x v+395$. (Academic: London and New York, 1976.) £14; $\$ 30.65$.

RECENT years have shown an upsurge of interest in the statistical mechanics of liquids, which has been marked by a number of important advances. The advance has been continuous since 1957; the previous history of the subject had been a series of fundamental advances, with long gaps in between.

This book is a timely and balanced survey of recent developments, shows how they are related to the older work, and explains the relationships between the various theoretical approaches. The year 1957 showed that the task of evaluating the correct consequences of an assumed intermolecular interaction function was within the power of large computers and this has stimulated a great deal of further work. One good feature is a special chapter describing techniques of these computer calculations.

The days of analytical results in this field are, however, very far from over, and these approaches are described in chapters 4, 5 and 6 . Basically what has happened is that the fundamental advances made by Kirkwood (the feasibility of an integral equation approach) and by Mayers (the diagram expansion technique) have been systematically followed up and improved. It has also been found possible to develop perturbation theories analogous to those of quantum mechanics and celestial mechanics. These three chapters provide a connected account of these developments, contain very little repetition and are a pleasure to read.

Some workers consider equilibrium statistical mechanics to be difficult to page headings; instead of the verso heading throughout the book unnecessarily proclaiming that it deals with "Sedum of North America", it could more usefully have given the chapter heading, while the recto heading then named the species dealt with beneath, which is often not evident. One hopes that some day Professor Clausen will produce a convenient illustrated handbook to the genus Sedum in America as a whole, summarising concisely the results of his many years of painstaking enquiry.

W. T. Stearn

W. T. Stearn is Honorary Botanical Curator of the Linnean Society, and was formerly a Senior Principal Scientific Officer at the British Museum (National History).

enough and fight shy of transport processes. A large amount of work has, however, been carried out, and is well summarised in chapters 7,8 and 9 , which the reviewer, largely an 'equilibrium man', found most helpful. In reading a published paper in this field it can be quite difficult to separate the formalism and the 'intelligent guesswork' or to see whether any difficulties of principle are being glossed over. These chapters will certainly be helpful to many workers, as will the account of generalised hydrodynamics, hard to find elsewhere except in original papers.

I was disappointed to see no mention of surface tension and related phenomena. One of the first definite results in liquid-state theory was Laplace's relation between surface tension and latent heat, and the subject was again opened up by the pioneer work of Kirkwood and Buff. Many of the published papers are, however, obscure and complicated; perhaps lack of space was the deciding factor. The final chapter, on phase transitions, seems to have suffered for a similar reason. The discussion of melting is as good as could have been hoped for in the present rather fragmentary state of knowledge but I would have liked to see a far more extensive account of the critical region. In particular, I would have liked to know the authors' own views on the following point. Why do scaling and renormalisation group methods provide good results despite the fact that they seem to use approximations just as drastic as those that vitiated older theories of phase transitions?

The book is well printed and produced but the price seems on the high side even at present levels. There is a valuable bibliography and a good index.

H. N. V. Temperley

H. N.V. Temperley is Professor of Applied Mathematics at University College of Swansea, University of Wales, UK 